\title{
Short Pulse Width and High Peak Power 457 nm Deep Blue Laser with V-Type Cavity
}

\author{
Zhibin Zhao',2, Cheng Cheng², Hao Chen², Quan $\mathrm{Li}^{2}$, Yi Qu ${ }^{2 *}$, Guojun Liu², Zhongliang Qiao², \\ Baoxue Bo ${ }^{*}$
}

${ }^{1}$ College of Opto-Electronic Engineering, Changchun University of Science and Technology, Changchun, China

${ }^{2}$ Key Laboratory of Laser Technology and Optoelectronic Functional Materials of Hainan Province, College of Physics and Electronic Engineering, Hainan Normal University, Haikou, China

Email: *quyihainan@126.com, ^bbx@cust.edu.cn

How to cite this paper: Zhao, Z.B., Cheng, C., Chen, H., Li, Q., Qu, Y., Liu, G.J., Qiao, Z.L. and Bo, B.X. (2021) Short Pulse Width and High Peak Power $457 \mathrm{~nm}$ Deep Blue Laser with V-Type Cavity. Journal of Applied Mathematics and Physics, 9, 2987-2994. https://doi.org/10.4236/jamp.2021.911192

Received: September 28, 2021 Accepted: November 27, 2021 Published: November 30, 2021

\begin{abstract}
An intracavity frequency doubling acousto-optically Q-switched Neodymium-doped Yttrium Orthvanadate $\left(\mathrm{Nd}: \mathrm{YVO}_{4}\right) 457 \mathrm{~nm}$ blue laser by employing a three-mirror folded cavity was demonstrated. With the incident pump power of $40.4 \mathrm{~W}$, the maximum average output power of $439 \mathrm{~mW} 457$ $\mathrm{nm}$ laser, and the minimum pulse duration of $86.14 \mathrm{~ns}$ and the maximum peak power of $510 \mathrm{~W}$ were achieved at $10 \mathrm{kHz}$. The $\mathrm{M}^{2}$ factors are 1.23 and 1.61 in $\mathrm{X}$ and $\mathrm{Y}$ directions, respectively. The power stability in two hours is better than $2 \%$.
\end{abstract}

\section{Keywords}

Laser, All-Solid-State Laser, Pulse Wave, Deep Blue Laser, 457 nm Laser

\section{Introduction}

All-solid-state blue and green lasers have a wide range of applications in ocean exploration systems, such as laser depth sounding, laser underwater communication, underwater target detecting, and seawater optical properties detecting [1] [2] [3]. Up to now, high peak power neodymium-doped all-solid-state $473 \mathrm{~nm}$ and $486.1 \mathrm{~nm}$ lasers have made great progress [4] [5]. However, the researches of all-solid-state deep blue lasers at $457 \mathrm{~nm}, 456 \mathrm{~nm}$ and $453 \mathrm{~nm}$ were relatively weak [6] [7] [8]. Below the thermocline, the transmission window of seawater is 430 - $470 \mathrm{~nm}$ [5]. Therefore, compared with the $473 \mathrm{~nm}$ and $486.1 \mathrm{~nm}$ laser, the $457 \mathrm{~nm}$ laser is more suitable for deep seawater detection. As we know, frequency doubling from a $0.9 \mu \mathrm{m}$ quasi three-level transition of neodymium-doped laser gain medium is a common method to get a deep blue laser. In the past, many 
researchers focused on developing $\mathrm{Nd}: \mathrm{YVO}_{4} / \mathrm{LBO}$ continuous-wave blue lasers [6] [9] [10], which are applicable for color displays, flow cytometry, and highdensity optical data storage. But for deep-seawater research, the high peak power and the short pulse width and the high repetition rate of the pulsed deep blue laser will help to improve its transmission distance and speed. So far, there are only a few reports on pulsed $457 \mathrm{~nm}$ lasers. Gao et al. [11] used a four-mirror-folded Z-shaped cavity; the pulse duration of $217 \mathrm{~ns}$ and the peak power of $258 \mathrm{~W}$ of $457 \mathrm{~nm}$ laser were achieved at $10 \mathrm{kHz}$. It can be seen that the laser cavity length is too long using Z-shaped cavity, laser pulses show wide pulse duration and therefore low peak power could be obtained at high-repetition rate operation. Some of the above problems can be avoided by using a three-mirror-folded V-shaped cavity.

In this paper, a three-mirror-folded V-shaped cavity was adopted in the pulsed laser system at $457 \mathrm{~nm}$, using an $808 \mathrm{~nm}$ laser diode (LD) to pump the $\mathrm{Nd}: \mathrm{YVO}_{4}$ laser crystal, through acousto-optically Q-switched and intracavity frequency doubling, with the incident pump power of $40.4 \mathrm{~W}$, the maximum average power of $439 \mathrm{~mW} 457 \mathrm{~nm}$ laser was achieved at $10 \mathrm{kHz}$, with the pulse duration of $86.14 \mathrm{~ns}$ and the peak power of $510 \mathrm{~W}$. To the best of our knowledge, this is the shortest pulse width and the highest peak power of the $457 \mathrm{~nm}$ deep blue laser so far.

\section{Cavity Parameter Optimization}

In this experiment, a three-mirror folded cavity was introduced to reduce the whole length of the cavity and to produce two separate beam waists. One is aimed at improving the output power of the fundamental frequency laser, the other is for high efficiency frequency doubling in LBO. According to the ABCD matrix theory and the stability conditions of the thermally insensitive cavity, the parameters of the resonator were optimized by MATLAB simulation calculation. Experimental setup is shown in Figure 1. Figure 2 and Figure 3 show the influence of the lengths of arm L1 and L2 on the stability of the cavity. It is obvious that different thermal lens focal lengths do not affect the stable region of the cavity. When the length of L1 changes by $20 \mathrm{~mm}$, the stability parameter only changes less than 0.1 , so the stability of the cavity is insensitive to the length of arm L1. However, we should notice that a $10 \mathrm{~mm}$ change of the second arm L2

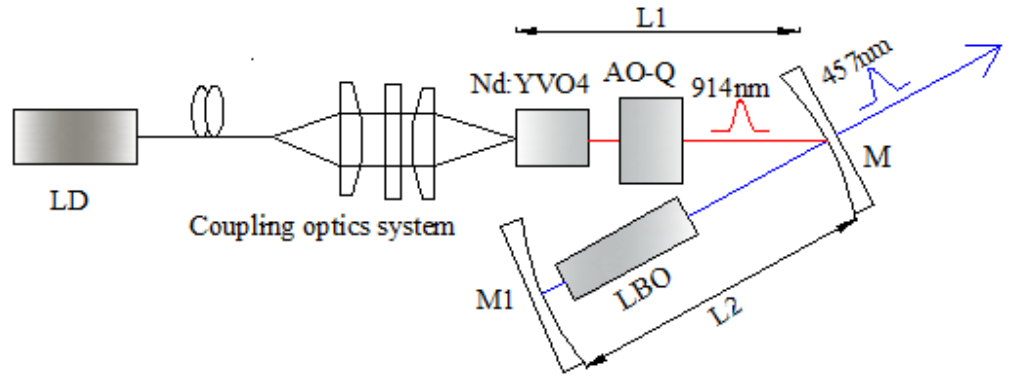

Figure 1. Experimental setup. 


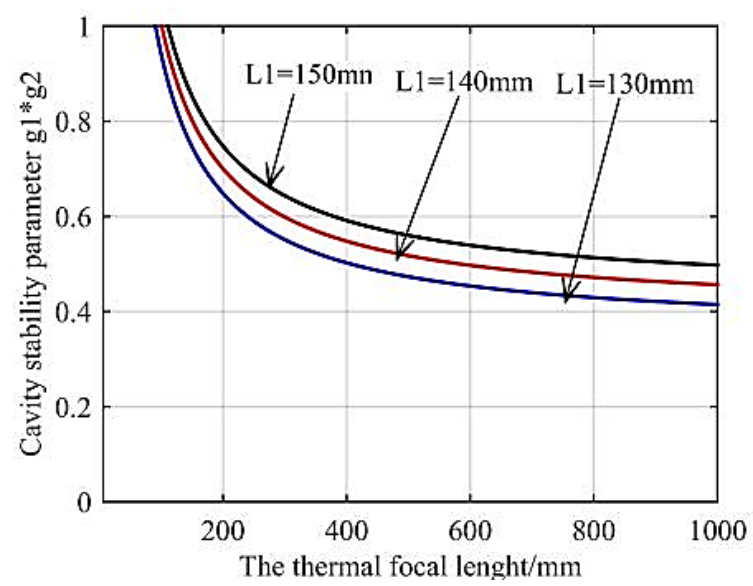

Figure 2. Influence of the length of arm L1 on the stability of the cavity.

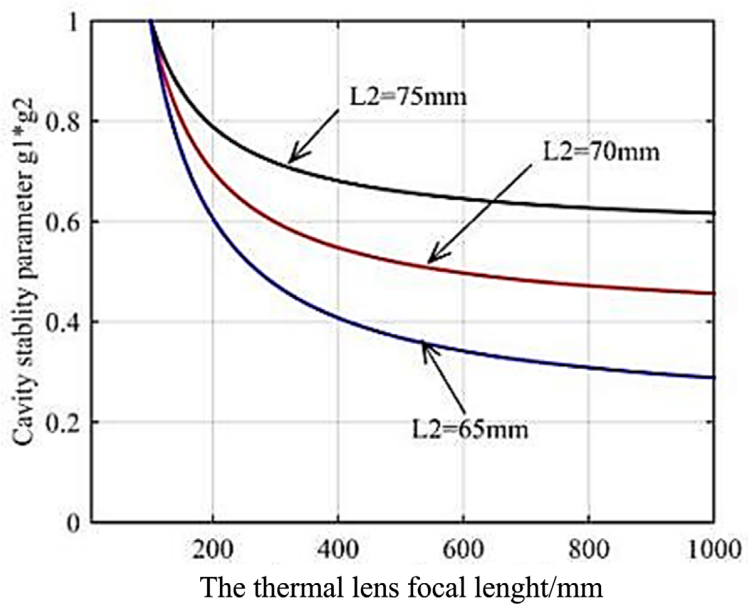

Figure 3. Influence of the length of arm L2 on the stability of the cavity.

will change the cavity stability parameter by 0.3 . Therefore the length of L2 must be adjusted carefully in the experiment. In addition, it can also be seen that the stability parameter of the cavity is closest to 0.5 with $\mathrm{L} 1=140 \mathrm{~mm}$ and $\mathrm{L} 2=70$ $\mathrm{mm}$.

\section{Experiment Setup}

Experimental setup is shown in Figure 1. The pump source is a $110 \mathrm{~W} 808 \mathrm{~nm}$ fiber-coupled LD with a core diameter of $400 \mu \mathrm{m}$ and a numerical aperture of 0.22 for continuous pumping. Its emission central wavelength is $806 \mathrm{~nm}$ at room temperature and can be tuned by changing the temperature of the heat sink to match the absorption of the laser crystal. Its temperature was kept at $27^{\circ} \mathrm{C}$ in the experiment to match the absorption peak of the laser crystal. The coupling optics consists of two identical plano-convex lenses with focal lengths of $12 \mathrm{~mm}$ and a polarizer used to re-image the pump beam into the laser crystal. The focused pump beam waist radius is about $250 \mu \mathrm{m}$, and its position in the laser crystal is about $1 \mathrm{~mm}$ away from the pump surface. The small pump beam ra- 
dius is used to mode matching. A Nd: $\mathrm{YVO}_{4}$ crystal with a doping-concentration of 0.1 at $\%$ and the dimensions of $4 \times 4 \times 5 \mathrm{~mm}^{3}$ was employed as a gain medium. The Nd: $\mathrm{YVO}_{4}$ crystal wrapped with a $0.1 \mathrm{~mm}$ thick indium foil is mounted on a copper micro-channel heat sink, and is cooled by water at a temperature of $26^{\circ} \mathrm{C}$. The left side of the laser crystal is coated with high-reflection (HR) film at $914 \mathrm{~nm}(\mathrm{R}>99.8 \%)$ and with antireflection (AR) film at $808 \mathrm{~nm}$ ( $\mathrm{T}>99 \%$ ), acting as one mirror of the cavity. The right-hand side of the laser crystal is AR coated at $914 \mathrm{~nm}(\mathrm{R}<0.2 \%)$ to reduce loss of the resonating 914 $\mathrm{nm}$ oscillation, and for suppressing the strong lines of $1064 \mathrm{~nm}$ and $1342 \mathrm{~nm}$, it is also AR coated at $1064 \mathrm{~nm}(\mathrm{R}<2 \%)$ and $1342 \mathrm{~nm}(\mathrm{R}<10 \%)$. A short laser crystal with low-doped concentration is used to minimize thermal lensing and the reabsorption of quasithree-level emission while guaranteeing that enough pump energy will be absorbed. The radii of the concave face are $100 \mathrm{~mm}$ and 200 $\mathrm{mm}$ for folding mirror $\mathrm{M}$ and reflector $\mathrm{M} 2$ respectively. $\mathrm{M}$ has $\mathrm{HR}$ coating at $914 \mathrm{~nm}$, and high transmission (HT) coating at $1064 \mathrm{~nm}$ and $1342 \mathrm{~nm}$, and partially at $457 \mathrm{~nm}$. M1 has HR coating at $914 \mathrm{~nm}$ and $457 \mathrm{~nm}$. An acousto-optical (A-O) Q-switch of quartz crystal (Gooch \& Housego Company) with central wavelength at $914 \mathrm{~nm}$ is placed close to the laser crystal in the L1 arm. Its maximum radio frequency power is $20 \mathrm{~W}$ and the effective aperture is $2.0 \mathrm{~mm}$. A LBO crystal with type-I phase matching $\left(\theta=90^{\circ}, \varphi=21.7^{\circ}\right)$ and the dimensions of $4 \times 4 \times 15 \mathrm{~mm}^{3}$ is employed as the frequency-doubler. Both sides of the LBO are AR coated at $914 \mathrm{~nm}$ and $457 \mathrm{~nm}$ and $1064 \mathrm{~nm}$. It is wrapped with a $0.1 \mathrm{~mm}$ thick indium foil and mounted on a copper micro-channel heat sink, which is cooled by water at a temperature of $26^{\circ} \mathrm{C}$. It is placed close to $\mathrm{M} 1$ in the $\mathrm{L} 1 \mathrm{arm}$ to obtain high frequency doubling efficiency. The length of LBO could be used to compensate the relatively smaller value of the nonlinear coefficient. The average output power of laser is measured by an optic power-meter (NOVA II-Israeli Ophir Inc.). The Q-switched pulses were detected by a Si Detector (DET10A/M-Thorlabs Inc.) and were recorded by a digital storage oscillograph (TDS 3054C-Tektronix Inc.). The beam quality of laser is measured by Beam quality analyzer, made in Israeli Ophir Company (BP209/VIS-Thorlabs Inc.).

\section{Results and Discussions}

At $10 \mathrm{kHz}$, the average output power and pulse width of the $457 \mathrm{~nm}$ deep laser as a function of the incident pump power are presented in Figure 4. It can be seen that the average output power is increased and the pulse width is reduced exponentially with the increase of the incident pump power. However, the relationship between average output power and pumping power is not linear. When the pump power is less than a certain level, the slope efficiency of the $457 \mathrm{~nm}$ average output power is very low, whereas, when the pump power is higher than that level, the slope efficiency increases dramatically. The reason for this phenomenon is caused by the saturation of the re-absorption loss of the quasi-three-level laser system. At the lower pump level, the population inversion and fundamental 
gain are very small, and the re-absorption loss will lead to relatively low slope efficiency, as the pump power increased, the fundamental gain is large enough to offset the impact of re-absorption. When the incident pump power is $40.4 \mathrm{~W}$, the maximum average power of $439 \mathrm{~mW}$, and the minimum pulse duration of $86.14 \mathrm{~ns}$ and the peak power of $510 \mathrm{~W}$ for $457 \mathrm{~nm}$ laser were achieved. Figure 5 and Figure 6 show the pulse trains and the typical pulse profile of the $457 \mathrm{~nm}$ deep blue laser at the maximum average output power $(439 \mathrm{~mW})$. Here the lost pulse and the double pulses phenomenon are not observed.

Laser beam intensity profile of the pulsed $457 \mathrm{~nm}$ deep blue laser at the maximum average output power of $439 \mathrm{~mW}$ is shown in Figure 7. It can be seen that the laser intensity distribution is very symmetrical and near Gaussian-distribution. Beam quality is measured by using a traveling knife-edge method, and $\mathrm{M}^{2}$ factors are estimated to be $\mathrm{M}_{\mathrm{x}}{ }^{2}=1.23$ and $\mathrm{M}_{\mathrm{y}}{ }^{2}=1.61$. The beam spot has an elliptical profile, which is attributed to the fact that the frequency-doubling crystal LBO used in the experiment is type-I phase- matching, and there is a walk-off along the polarization direction of the e-ray.

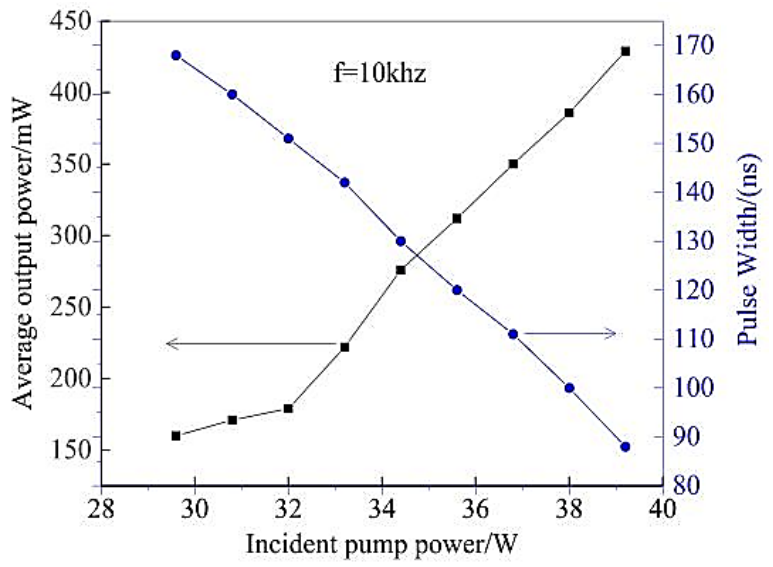

Figure 4. Average output power and pulse width of the $457 \mathrm{~nm}$ deep blue laser VS pump power at $10 \mathrm{kHz}$.

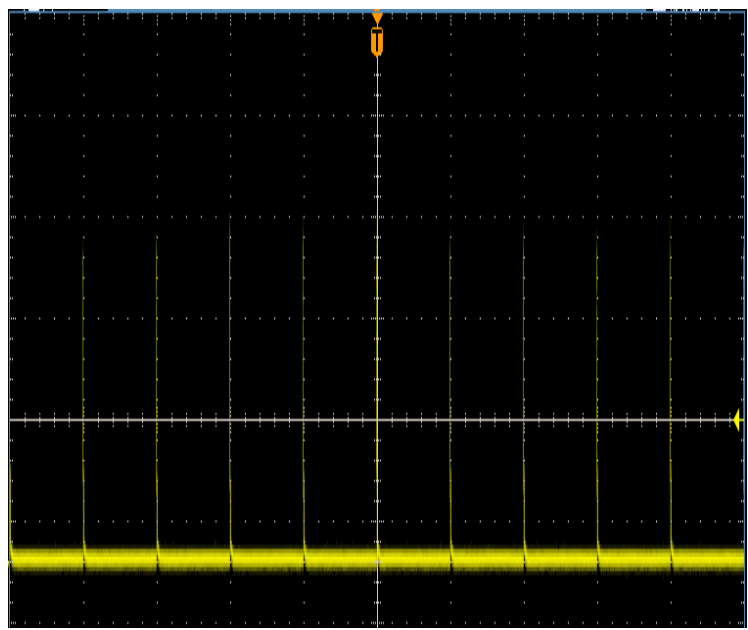

Figure 5. The pulse trains of the $457 \mathrm{~nm}$ deep blue laser. 
The power stability of the pulsed $457 \mathrm{~nm}$ deep blue laser was investigated. Figure 8 shows the time trace of the maximum average output power of the pulsed $457 \mathrm{~nm}$ deep blue laser for two hours. The fluctuation of the output power for the pulsed $457 \mathrm{~nm}$ deep blue laser is less than $2 \%$. The good power stability was attributed to the precise temperature control in $\mathrm{Nd}: \mathrm{YVO}_{4}$ and LBO crystal. On one hand, little temperature fluctuation of the laser medium results in a

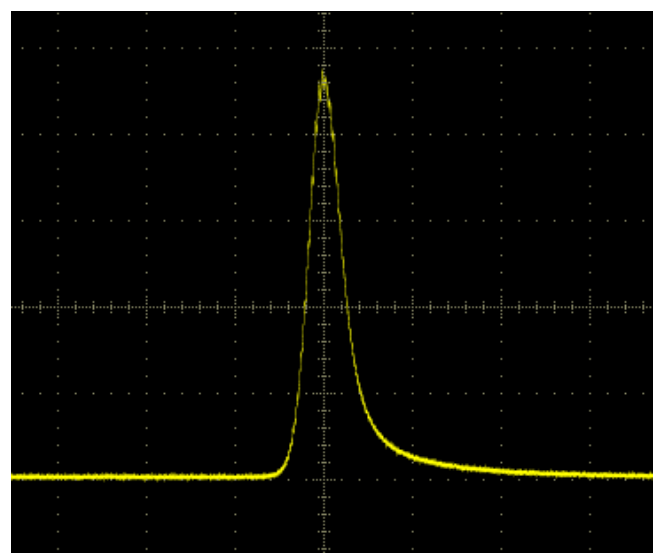

Figure 6. The pulse profile of the $457 \mathrm{~nm}$ deep blue laser.

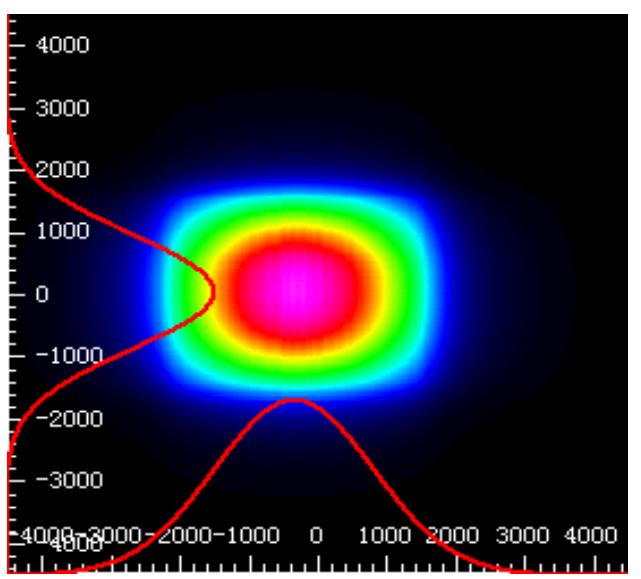

Figure 7. Laser intensity profile of the pulsed $457 \mathrm{~nm}$ deep blue laser.

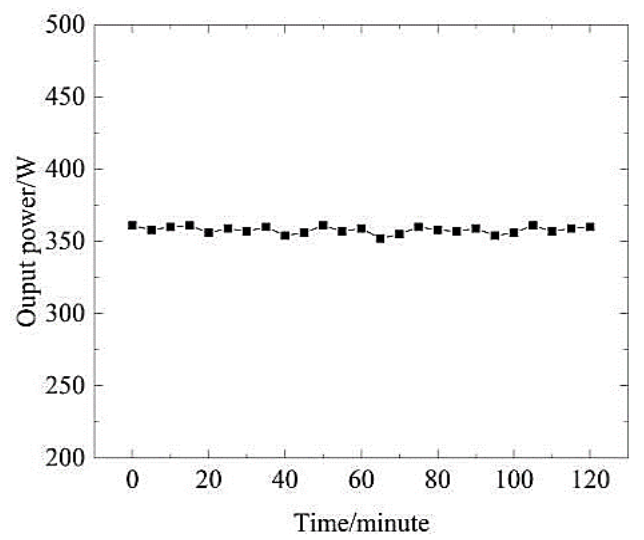

Figure 8. The $457 \mathrm{~nm}$ deep blue laser power stability. 
minor change of the thermal population on the lower level, which enhances the output power stability of the fundamental laser. On the other hand, small temperature changes in the nonlinear crystal lead to accurate phase-match between the $914 \mathrm{~nm}$ fundamental laser and the $457 \mathrm{~nm}$ second harmonic laser.

\section{Conclusion}

We have demonstrated an intracavity frequency doubling acousto-optically Q-switched $\mathrm{Nd}: \mathrm{YVO}_{4} 457 \mathrm{~nm}$ blue laser by employing a three-mirror folded cavity for the first time. A three-mirror folded cavity is employed to enhance the conversion efficiency. With an incident pump power of $40.4 \mathrm{~W}$, the maximum average power of $439 \mathrm{~mW} 457 \mathrm{~nm}$ laser was achieved at $10 \mathrm{kHz}$, with the minimum pulse duration of $86.14 \mathrm{~ns}$ and the maximum peak power of $510 \mathrm{~W}$. The $\mathrm{M}^{2}$ factors are 1.23 and 1.61 in $\mathrm{X}$ and $\mathrm{Y}$ directions, respectively. The power stability in two hours is better than $2 \%$.

\section{Acknowledgements}

This work was supported in part by Major Science and Technology Program of Hainan Province of China under Grant ZDKJ2019005; in part by the Hainan Provincial Natural Science Foundation of China under Grant 618QN241; in part by the Young Talents Science and Technology Innovation Project of Hainan Association for Science and Technology under Grant QCXM201810; in part by Special Research Project of Hainan Academician Innovation Platform under Grant SPTZX202034; in part by the National Natural Science Foundation of China under Grant 61774024, Grant 61864002, Grant 11764012, and Grant 61964007; in part by Key R \& D Projects in Hainan Province under Grant ZDYF2020020, Grant ZDYF2020036 and Grant ZDYF2020217; in part by Hainan Natural Science Foundation Innovation Research Team under Grant 2018CXTD336.

\section{Conflicts of Interest}

The authors declare no conflicts of interest regarding the publication of this paper.

\section{References}

[1] Wu, T.C., Chi, Y.C., Wang, H.Y., Tsai, C.T. and Lin, G.R. (2017) Blue Laser Diode Enables Underwater Communication at 12.4 Gbps. Sci. Rep., 7, Article No. 40480. https://doi.org/10.1038/srep40480

[2] Ma, J., Lu, T.T., He, Y., Jiang, Zh.Y., Hou, Ch.H., Li, K.P., Liu, F.H., Zhu, X.L. and Chen, W.B. (2020) Compact Dual-Wavelength Blue-Green Laser for Airborne Ocean Detection Lidar. Appl. Opt., 59, C87-C91. https://www.osapublishing.org/ao/abstract.cfm?URI=ao-59-10-C87 https://doi.org/10.1364/AO.382174

[3] Zha, B.T., Yuan, H.L. and Tan, Y.Y. (2019) Ranging Precision for Underwater Laser Proximity Pulsed Laser Target Detection. Opt. Commun., 431, 81-87. 
https://doi.org/10.1016/j.optcom.2018.09.003

[4] Zhang, J.L., Ma, J., Lu, T.T., Wang, J.L., Zhu, X.L. and Chen, W.B. (2021) 16.9 MW, Efficient $486.1 \mathrm{~nm}$ Blue Optical Parametric Oscillator Using Single BBO Crystal. Laser Phys. Lett., 18, Article No. 025001. https://doi.org/10.1088/1612-202X/abd3f9

[5] Lu, T.T., Ma, J., Zhu, X.L. and Chen, W.B. (2019) Highly Efficient Electro-Optically Q-Switched 473 nm Blue Laser. Chin. Opt. Lett., 17, Article No. 051405. https://doi.org/10.3788/COL201917.051405

[6] Yin, H., Zhu, S.Q., Chen, Zh.Q., He, Q., Li, A.M., Li, Zh., Liu, Y.M., Su, K., Zhang, G. and Chen, Y.H. (2016) Research on All-Solid-State Intracavity Frequency Doubling $457 \mathrm{~nm}$ Laser with LBO and BIBO Crystal. Optik, 127, 3862-3866. https://doi.org/10.1016/j.ijleo.2015.12.163

[7] Chen, F., Yu, X., Zhang, K., He, Y., Zheng, C.B., Wang, C.R. and Guo, J. (2015) Diode-Pumped Acousto-Optical Q-Switched $912 \mathrm{~nm} \mathrm{Nd:GdVO} 4$ Laser and ExtraCavity Frequency-Doubling of $456 \mathrm{~nm}$ Deep-Blue Light Emission. Opt. Laser Technol, 68, 36-40. https://doi.org/10.1016/j.optlastec.2014.11.007

[8] Wang, J., Pan, Y.H. and Huang, Y.F. (2017) Low-Pump Sum Frequency Generation of Frequency-Stabilized $453 \mathrm{~nm}$ Blue Laser for Photonic Quantum Interface. Chin. Opt. Lett., 15, Article No. 122701. https://doi.org/10.3788/COL201715.122701 https://www.osapublishing.org/col/abstract.cfm?URI=col-15-12-122701

[9] He, Q., Zhu, S.Q., Chen, Z.Q. and Wang, S.E. (2014) High Efficiency and Low Threshold Diode-End-Pumped Blue-Ray Laser at $457 \mathrm{~nm}$ Wavelength with Straight Type Cavity. Optik, 125, 3503-3506. https://doi.org/10.1016/j.ijleo.2014.01.061

[10] Zheng, Q., Yao, Y., Li, B., Qu, D.P. and Zhao, L. (2009) $13.2 \mathrm{~W}$ Laser-DiodePumped Nd: $\mathrm{YVO}_{4} / \mathrm{LBO}$ Blue Laser at 457 nm. J. Opt. Soc. Am. B., 26, 1238-1242. https://www.osapublishing.org/josab/abstract.cfm?URI=josab-26-6-1238 https://doi.org/10.1364/JOSAB.26.001238

[11] Gao, J., Yu, X., Chen, F., Li, X.D., Zhen, Zh., Yan, R.P., Yu, J.H. and Wang, Y.Zh. (2008) $457 \mathrm{~nm}$ Blue Laser Generation by Intracavity Frequency Doubling of Acousto-Optically Q-Switched Nd: $\mathrm{YVO}_{4}$ Laser. Acta Optica Sinaca, 28, 9-13. 